Revue Française de Civilisation Britannique

\title{
Bibliographie de Jacques Leruez
}

Bibliography of Work by Jacques Leruez

\section{Edwige Camp-Pietrain}

\section{(2) OpenEdition}

1 Journals

Édition électronique

URL : https://journals.openedition.org/rfcb/4799

DOI : $10.4000 / \mathrm{rfcb} .4799$

ISSN : 2429-4373

Éditeur

CRECIB - Centre de recherche et d'études en civilisation britannique

Référence électronique

Edwige Camp-Pietrain, «Bibliographie de Jacques Leruez », Revue Française de Civilisation Britannique [En ligne], XXIV-4 | 2019, mis en ligne le 25 novembre 2019, consulté le 28 juin 2022. URL : http:// journals.openedition.org/rfcb/4799; DOI : https://doi.org/10.4000/rfcb.4799

Ce document a été généré automatiquement le 29 septembre 2020.

\section{(c) $($ ) $\odot$ (8Y NO}

Revue française de civilisation britannique est mis à disposition selon les termes de la licence Creative Commons Attribution - Pas d'Utilisation Commerciale - Pas de Modification 4.0 International. 


\title{
Bibliographie de Jacques Leruez
}

Bibliography of Work by Jacques Leruez

\author{
Edwige Camp-Pietrain
}

\section{NOTE DE L'AUTEUR}

Cette bibliographie a été préparée avec le concours d'Elisabeth Chaigneau Leruez. Elle ne peut toutefois prétendre à l'exhaustivité, en particulier en ce qui concerne les publications diverses. En outre, Jacques Leruez était régulièrement invité à participer à des émissions de radio, par exemple sur France Culture (Les enjeux internationaux, 27 juin 2007, 4 février 2009, 30 janvier 2013) ou RFI (15 septembre 2014, 28 mai 2015, 28 décembre 2015, 21 avril 2016).

\section{Ouvrages}

1 Planification et politique en Grande-Bretagne 1945-1971, Paris, Presses de la FNSP, 1972, 314 p., ouvrage rédigé à partir de la thèse L'Idée de planification économique en GrandeBretagne. Développements politiques et institutionnels, Paris, Université Panthéon-Assas, $1971,650 \mathrm{p}$.

2 Economic Planning and Politics 1945-1974, Londres, Martin Robertson, 1975, 324 p., traduction de Planification et politique en Grande-Bretagne 1945-1971. Le Royaume-Uni, trente ans de difficultés, Paris, Hatier, 1979, 80 p. ; réédition 1982.

4 (avec Sergeant Jean-Claude et Toboul William) Les partis politiques britanniques: du bipartisme au multipartisme?, Paris, PUF, 1982, 256 p.

5 L'Écosse, une nation sans État, Lille, Presses universitaires de Lille, 1983.

6 Le Phénomène Thatcher, Bruxelles, éditions Complexe, 1991, 336 p.

7 Gouvernement et politique en Grande-Bretagne, Presses FNSP et Dalloz, 1989, 423 p. 
8

$$
\text { 2001, } 331 \text { p. }
$$

9 (avec Morère Pierre) L'Écosse contemporaine. Politique, société, économie, culture, Paris, Ophrys-Ploton, 1995.

10 L'Écosse, Vieille nation, Jeune État, Crozon, éditions Armeline, 2000, 344 p. ; édition mise à jour de L'Écosse, une nation sans État.

11 Thatcher, la dame de fer, Bruxelles, Versaille, 2012, 242 p.; édition mise à jour de Le Phénomène Thatcher.

\section{Directions de périodiques et d'ouvrages}

Le Thatchérisme. Doctrine et Action, Paris, La Documentation française, Notes et études documentaires, octobre 1984, $143 \mathrm{p}$.

la Serre Françoise et Wallace Helen) Les Politiques étrangères de la France et de la Grande-Bretagne depuis 1945. L'inévitable ajustement, Paris, Presses de la FNSP, 1990, 295 p.

14 (avec de la Serre Françoise et Wallace Helen) French and British Foreign Policies in Transition. The Challenges of Adjustment, New York, Oxford, Munich, Berg Publishers, 1990.

15 La Grande-Bretagne à la fin du XXe siècle. L'héritage du thatchérisme, Paris, La Documentation française, Notes et études documentaires, n4997, 1994, 161 p.

16 (avec Civardi Christian) «L'Écosse contemporaine : politique, société, culture », Revue française de civilisation britannique, vol. IX, n²2, 1997.

17 Londres et le monde. Stratèges et stratégies britanniques, Paris, Autrement, 2005, 147 p.

\section{Chapitres d'ouvrages}

(avec Surel Jeannine) La Grande-Bretagne tome 3. Les temps difficiles 1914-1977, François Bedarrida (dir.), collection d'histoire contemporaine, Paris, Hatier université, 1978.

«Macroeconomic Planning in Mixed Economies : the French and British Experience ", in Hayward J. et Narkeiwicz C. (dir.), Planning in Europe, Londres, Croom Helm, 1978, p. 26-52.

"Planning in an Overloaded Economy ", in Rose Richard (dir.), Challenge to Governance: Studies in Overloaded Polities, Londres, Sage, 1980, p. 53-71.

21 "Les Méandres de Londres ", in Béja Jean-Philippe (dir.), Hong-Kong 1997, fin de siècle, fin d'un monde?, Bruxelles, éditions Complexe, 1993, p. 47-60.

" Le Chef de l'État dans le débat républicain en Australie: du Gouverneur-Général au Président de la République ", in Pons Xavier et Smit Corinne (dir.), Le Débat républicain en Australie, Paris, Ellipses, 1997, p. 60-75.

23 «Le Dirigisme de guerre et ses conséquences: naissance d'un État semicorporatiste ? », in Frison Danièle (dir.), La Société anglaise en guerre septembre 1939-août 1945, Paris, Ellipses, 1998, p. 54-63.

Revue Française de Civilisation Britannique, XXIV-4 | 2019 
24 «Britain, France and Economic Planning in the 1960s - the Commissariat au Plan, Role Model or Counter Model?", in Chassaigne Philippe (dir.), Anglo-French relations 1898-1998 : from Fashoda to Jospin, Londres, Palgrave Macmillan, 2002. «D'un Référendum à l'autre. L'expérience écossaise », in Leydier Gilles (dir.), La Dévolution des pouvoirs à l'Écosse et au pays de Galles 1966-1999, Paris, Ellipses, 2006, p. 15-30.

\section{Articles dans des revues scientifiques}

26 «Le Conflit israélo-arabe et l'action des puissances: la Politique de la GrandeBretagne ", Revue française de science politique, vol. 19, n², avril 1969, p. 429-443.

«Guerres civiles et conflits transnationaux: Irlande du Nord: intégration ou sécession? ", Revue française de science politique, vol. 21, n 4, août 1971, p. 836-858.

28 « Y-a-t-il une issue en Irlande du Nord? », Revue de Défense Nationale, novembre 1972, p. 1668-1686.

29 "Actualité du problème ethnique en Grande-Bretagne", Revue française de science politique, vol. 23, n5, octobre 1973, p. 1080-1090.

30 «Le Malaise politique en Grande-Bretagne ", Études, n9, août-septembre 1974, p. 291-296.

31 «Syndicalisme et politique: les syndicats britanniques face au gouvernement conservateur 1970-1974 ", Revue française de science politique, vol. 25, n5, octobre 1975, p. 919-945.

32 "La Crise nord-irlandaise ", Revue française de science politique, vol. 26, n³, juin 1976, p. 535-567.

33 « Où en est la Grande-Bretagne? De la crise économique aux incertitudes politiques », Défense nationale, $33^{\mathrm{e}}$ année, février 1977, p. 41-55.

34 «Partis politiques et élections dans l'Écosse d'aujourd'hui», Revue française de science politique, vol. 28, $\mathrm{n}^{\circ} 3$, juin 1978, p. 508-536.

35 «La dévolution écossaise », Études, juillet 1978, p. 43-58.

36 (avec Charlot Monica et Brennan Paul) «Élections en Grande-Bretagne », Projet, juilletaoût 1979, p. 863-880.

37 «Régionalisme et politique au Royaume-Uni. Les Scrutins du $1^{\mathrm{er}}$ mars et du 3 mai 1979 en Écosse et l'échec de la dévolution ", Revue française de science politique, vol. 29, n6, décembre 1979, p. 1064-1089. «Le Cabinet-fantôme : l'opposition institutionnalisée en Grande-Bretagne », Pouvoirs, $\mathrm{n}^{\circ} 12$, février 1980, p. 165-178.

39 «Dévolution et politique au Royaume-Uni : l'État central et les nationalismes écossais et gallois ", Revue française de civilisation britannique, vol. II, n³, janvier 1984, p. 63-82.

40 «La Grande-Bretagne après les élections de juin 1983 ", Études, n¹, janvier 1984, p. 5-18.

41 "L'Enjeu de la Défense : l'“élection nucléaire” n'aura pas lieu », Revue française de civilisation britannique, 1984, p. 57-76. 

1962-1970 ", Revue française de civilisation britannique, vol. III, nº 1 , décembre 1984, p. 25-42.

53 "Bilan des privatisations sous les gouvernements Thatcher", Revue française de civilisation britannique, vol. VI, n4, février 1992, p. 29-41. "L'Écosse, l'État britannique et les élections générales du 9 avril 1992 : contribution à une réflexion sur les nations sans État d'Europe occidentale ", Pouvoirs, ${ }^{\circ} 63$, novembre 1992, p. 129-144.

«L'Élection du 9 avril 1992 en Écosse », Revue française de civilisation britannique, vol. VII, nº 1 , décembre 1992, p. 119-134.

"L'Hégémonie Tory. Les élections du 9 avril 1992 au Royaume-Uni : aboutissement heureux d'une "alternance douce" ", Commentaire, n59, automne 1992, p. 629-638.

«Lettres de Grande-Bretagne: le Royaume-Uni depuis les élections d'avril 1992 - le chemin de croix de John Major », Pouvoirs, n70, 1994, p. 163-172.

«Fin du règne conservateur au Royaume-Uni : les élections générales du $1^{\mathrm{er}}$ mai 1997 ", Pouvoirs, n83, novembre 1997, p. 165-175.

59 «Le nouveau Parlement écossais et les élections du 6 mai 1999 », Pouvoirs, n90, 1999, p. $153-164$

60 «L'Actualité de Beveridge. À propos de la réédition de la grande biographie de William Beveridge ", Vingtième Siècle. Revue d'histoire, n¹, 1999.

"L'Écosse vers l'autonomie politique ", Vingtième Siècle. Revue d'histoire, n65, janviermars 2000, p. 109-123. 
62 «La "Relation Spéciale" sous Margaret Thatcher (1979-1990)», Revue française de civilisation britannique, vol. XII, n²1, décembre 2002, p. 101-112. en Écosse », Pouvoirs, n¹07, 2003, p. 145-159.

65

\section{Divers}

\section{Encyclopédie Universalis}

« Royaume-Uni : vie et institutions politiques », p. 708-720.

«Grande-Bretagne : l'année des deux élections », Universalia, 1975, p. 293-296.

(avec Burgi Noëlle) « Grande-Bretagne : le conflit des charbonnages », Universalia, 1986, p. 260-263.

« Où va le thatchérisme ? », Universalia, 1987, p. 281-284.

«Grande-Bretagne. Où en sont les privatisations ? ", Universalia, 1990, p. 262-265.

«Grande-Bretagne : le départ de Mme Thatcher », Universalia, 1991, p. 282-284.

"Grande-Bretagne. La Division Nord-Sud: historique et réalités contemporaines », Universalia, 1992, p. 274-277.

« Grande-Bretagne. Une élection pour rien ? ", Universalia, 1993, p. 244-246.

"Grande-Bretagne. Vers un nouveau Labour », Universalia, 1995.

«Grande-Bretagne. De John Major à Tony Blair. Les élections générales du $1^{\mathrm{er}}$ mai 1997», Universalia, 1998.

«Grande-Bretagne. Tony Blair. Mauvaise passe ou réel déclin ?», Universalia, 2006, p. 229-232.

http://www.universalis-edu.com/encyclopedie/tony-blair

http://www.universalis-edu.com/encyclopedie/james-callaghan

http://www.universalis-edu.com/encyclopedie/edward-heath

http://www.universalis-edu.com/encyclopedie/john-major 


\section{La Documentation française} 1975 ", Problèmes politiques et sociaux, n²65, Paris, La Documentation française, août 1975, 44 p.

Le Royaume-Uni. L'année Thatcher ", Les Pays d'Europe occidentale. Notes et études documentaires, $\mathrm{n}^{\circ} 4565$, Paris, La Documentation française, avril 1980, p. 13-33.

91 «Le Royaume-Uni en 1980 », Les Pays d'Europe occidentale. Notes et études documentaires, $\mathrm{n}^{\circ}$ 4621, Paris, La Documentation française, mai 1981, p. 13-29.

92 « Le Royaume-Uni en 1981 », Les Pays d'Europe occidentale. Notes et études documentaires, $\mathrm{n}^{\circ}$ 4661, Paris, La Documentation française, mars 1982, p. 15-41. «1982 au Royaume-Uni : l'année des Falkland», Les Pays d'Europe occidentale. Notes et études documentaires, $\mathrm{n}^{\circ}$ 4723, Paris, La Documentation française, juin 1983, p. 13-48.

94 "Le Royaume-Uni en 1983 », Les Pays d'Europe occidentale. Notes et études documentaires, $\mathrm{n}^{\circ}$ 4760, Paris, La Documentation française, 1984, p. 13-42.

95 "Le Thatchérisme. Doctrine et Action ", Les Pays d'Europe occidentale. Notes et études documentaires, $\mathrm{n}^{\circ} 4765$, Paris, la documentation française, 1984, $144 \mathrm{p}$.

96 « Le Royaume-Uni en 1984 », Les Pays d'Europe occidentale. Notes et études documentaires, $\mathrm{n}^{\circ}$ 4783, Paris, La Documentation française, 1985, p. 13-40.

97 «Le Royaume-Uni au début de 1986, le gouvernement Thatcher devant la montée des périls", Les Pays d'Europe occidentale. Notes et études documentaires, $n^{\circ} 4813$, Paris, la documentation française, septembre 1986, p. 13-36. «Le Royaume-Uni en 1987 : troisième mandat pour Mme Thatcher ", Les Pays d'Europe occidentale. Notes et études documentaires, Paris, La Documentation française, septembre 1988, p. 15-42. «Le Royaume-Uni en 1988 : la deuxième vague radicale », Les Pays d'Europe occidentale. Notes et études documentaires, Paris, La Documentation française, septembre 1989, p. 15-34.

100 «Le Royaume-Uni en 1989: Mme Thatcher face à une crise de confiance", Les Pays d'Europe occidentale. Notes et études documentaires, Paris, La Documentation française, septembre 1990, p. 15-36.

101 «Le Royaume-Uni en 1990 », Les Pays d'Europe occidentale. Notes et études documentaires, Paris, La Documentation française, septembre 1991, p. 17-39.

102 «Le Royaume-Uni en 1991. Une succession délicate », Les Pays d'Europe occidentale. Notes et études documentaires, Paris, La Documentation française, septembre 1992, p. 15-39.

103 «Le Royaume-Uni en 1992. Une année "exécrable"?», Les Pays d'Europe occidentale. Notes et études documentaires, Paris, La Documentation française, 1993, p. 19-40. 
104 « Le Royaume-Uni en 1998 : un “état de grâce” persistant », Les Pays d'Europe occidentale. Notes et études documentaires, Paris, La Documentation française, 1999.

"Les Institutions Politiques de la Grande-Bretagne. Documents réunis et commentés », Documents d'études. Droit constitutionnel et institutions politiques, 1.03, Paris, La Documentation française, 1989, 1994, 40 p.; «Les Institutions Politiques du RoyaumeUni », 1999, $51 \mathrm{p}$.

106 «Le Système politique britannique: que reste-t-il du modèle de Westminster?", Questions internationales, $n^{\circ} 66$, Paris, La Documentation française, juillet-août 2006.

107 "L'Écosse à l'heure du choix », Questions internationales, n66, Paris, La Documentation française, mars-avril 2014.

\section{Autres}

108 «Programmes et difficultés du gouvernement travailliste. M. Wilson se heurte aux faiblesses structurelles de l'économie », Le Monde Diplomatique, avril 1974.

109 (avec de la Serre Françoise) «Le Référendum européen en Grande-Bretagne : victoire des modérés et clivage au sein des partis », Le Monde Diplomatique, juillet 1975.

110 (avec Charlot Monica) « Deux tests pour la régionalisation au Royaume-Uni : en Écosse, une réponse à la normande. Au pays de Galles, un non "franc et massif", Le Monde diplomatique, avril 1979.

111 « Royaume-Uni : jusqu'où aller trop loin? », Le Figaro, 25-26 septembre 1982, p. 13-14.

112 «Un Exemple de nationalisme: le parti national écossais SNP », Revue Historique Bretonne, $\mathrm{n}^{\circ}$ 10, janvier 1985, p. 10-16.

113 Margaret Thatcher et l'Écosse, Paris, Association franco-écossaise, hors-série, avril 1993.

114 (avec Dickson George) L'Écosse contemporaine, Actes du colloque franco-écossais tenu au Sénat le 18 septembre 1996 à l'occasion du centenaire de l'association franco-écossaise, Paris, $1998,59 \mathrm{p}$.

115 Auld Alliances, New Alliances: Scotland, Europe and France Toward the Next Century, Édimbourg, Franco-Scottish Society of Scotland.

116 (sous la direction de), Où va l'Écosse après 13 ans de dévolution?, Actes du colloque de l'association franco-écossaise, Paris, association franco-écossaise, 2012, $50 \mathrm{p}$.

117 « Royaume-Uni : enjeux », in Généreux Jacques (dir.), Enjeux du Monde, Paris, Hachette, 1988, p. 241-249.

118 «Grande-Bretagne. Difficultés à l'intérieur, immobilisme à l'extérieur ", L'Année internationale, 1990-1991, Paris, Hachette, 1990, p. 36-38.

119 Le Royaume-Uni après les élections de mai 1997 ; Changement de gouvernement ou changement de régime, Paris, Études du CERI, n³8, janvier 1998, 45 p.

\section{Recensions}

120 «Parkin Frank, Radicalism, the Socialist Bases of the British Campaign for Nuclear Disarmament; Blackburn Robin, Cockburn Alexander (dir.). The Incompatibles. Trade Union Militancy and the Consensus ", Revue française de science politique, vol. 19, n³, 1969, p. 708-711. 
121 «Fort Paul, The Politics of Harold Wilson ", Revue française de science politique, vol. 19, n6, 1969, p. 1260-1262.

« Hirschman Albert Otto, Exit, Voice and Loyalty Responses to Decline in Firms, Organisations and States ", Revue française de science politique, vol. 22, n4, 1972, p. 914-916.

"Barnett Correlli, The Collapse of British Power ", Revue française de science politique, vol. 23, nº 4, 1973, p. 873-876.

124 «Raymond Vernon, Les entreprises multinationales. La souveraineté nationale en péril; Hugh Stephen, The Coming Clash. The Impact of the National Corporation on the Nation State", Revue française de science politique, vol. 24, $\mathrm{n}^{\circ} 1,1974$, p. 127-129.

"Fitzgerald Garret, Towards New Ireland; O'Brien Conor, States of Ireland», Revue française de science politique, vol. 24, n¹, 1974, p. 129-132.

126 «Heclo Hugh, Wildarsky Aaron, The Private Government of Public Money : Community and Policy Inside British Politics ", Revue française de science politique, vol. 25, n², 1975, p. 342-345.

127 « Bedarida François, La société anglaise 1851-1975 », Revue française de science politique, vol. $26, \mathrm{n}^{\circ} 6,1976$, p. $1158-1160$.

"Stewart Michael, The Jekyll and Hyde Years. Politics and Economic Policy since 1964 », Revue française de science politique, vol. 28, $\mathrm{n}^{\circ} 1,1978$, p. 148-149.

"Chester Norman, The Nationalisation of British Industry 1945-1951", Revue française de science politique, vol. 28, n5, 1978, p. 925-927.

130 «Butler David, Kitzinger Uwe, The 1975 Referendum; Goodhart Philip, Full-Hearted Consent. The Story of the Referendum Campaign and the Campaign for the Referendum ; King Anthony, Britain Says Yes. The 1975 Referendum on the Common Market ", Revue française de science politique, vol. 28, $\mathrm{n}^{\circ} 6,1978$, p. 1121-1122.

131 «Butler D., Ranney Austin (dir.), Referendums. A Comparative Study of Practice and Theory ", Revue française de science politique, vol. 29, n³, p. 511-513.

132 "Middlemas Keith, Politics in Industrial Society: the Experience of the British System since 1911 ; Alt James, The Politics of Economic Decline. Economic Management and Behaviour in Britain since 1964 ", Revue française de science politique, vol. 30, n6, 1980, p. 1313-1315.

133 "Charlot Monica, Élections de crise en Grande-Bretagne», Revue française de science politique, vol. 30, n¹, 1980, p. 148-149.

134 «Williams Raymond, Politicians and Letters, Interviews with New Left Review; Wright AW, GDH Cole and Social Democracy", Revue française de science politique, vol. $31, \mathrm{n}^{\circ} 1,1981, \mathrm{p}$. 268-270.

135 «Williams Philip, Hugh Gaitskell. A Political Biography », Revue française de science politique, vol. 31, n³, 1981, p. 593-595.

136 "Ravier Jean-Pierre, Les Syndicats britanniques sous les gouvernements travaillistes", Revue française de science politique, vol. 32, n² 2, 1982, p. 283-284. "Les Séquelles du débat sur la dévolution en Grande-Bretagne. Notes de recherches bibliographiques ", Revue française de science politique, vol. 32, n 4-5, 1982, p. 860-864.

"Kermode D., Devolution at Work, Case Study of the Isle of Man ", Revue française de science politique, vol. 32, n4-5, 1982, p. 865-866. 
139 «Harris Kenneth, Attlee », Revue française de science politique, vol. 33, n5, 1983, p. 888-890. «Borthwisk RL., Spenge JE. (dir.), British Politics in Perspective; Budge I., MacKay D. et al., The New British Political System. Government and Society in the 1980s; Leys C. , Politics in Britain. An Introduction ", Revue française de science politique, vol. 35, n¹, 1985, p. 119-122. "Tiryakian Edward, Rogowski Ronald (dir.), «New Nationalisms of the Developed West. Towards Explanations; Meny Y, Wright V. (dir.), Centre-Periphery Relations in Western Europe ; Keating M., Jones B (dir.), Regions in the European Community ", Revue française de science politique, vol. 36, $\mathrm{n}^{\circ} 5,1986, \mathrm{p} .698-701$. «Hayward Jack, The State and the Market Economy. Industrial Patriotism and Economic Intervention in France ", Revue française de science politique, vol. 37, $\mathrm{n}^{\circ} 1,1987, \mathrm{p}$. 99-101. "Jowell Jeffrey, Dawn Oliver (dir.), The Changing Constitution; Marshall Geoffrey, Constitutional Conventions. The Rules and Forms of Political Accountability; Journès Claude, L'État britannique; Jones Barry, Keating Michael, Labour and the British State», Revue française de science politique vol. 37, n6, 1987, p. 916-919.

144 «Goehlert Robert, Martin Fenton, The Parliament of Great Britain : a Bibliography; Judge David, The Politics of Parliamentary Reform ; Norton Philip (dir.), Parliament in the 1980s; Jones Barry J., Wilford RA. ", The Committee on Welsh Affairs ", Revue française de science politique, vol. $38, \mathrm{n}^{\circ} 1,1988$, p. 138. "Lewis J., Townsend A., The North-South Divide. Regional Change in Britain in the 1980s; Smith D., North and South, Britain's Growing Divide; Osmond J., The Divided Kingdom; Johnston RJ., Pattie CJ., Allsopp JG., A Nation Dividing? The Electoral Map of Great Britain 1979-1987 », Revue française de science politique, vol. 40, n², 1990, p. 276-279.

146 "Bergougnioux A., Manin B., Le Régime social-démocrate ", Revue française de science politique, vol. 40, n4, 1990, p. 623-627.

147 "Le Thatchérisme revisité ", Revue française de science politique, vol. 41, n5, 1991, p. 693-699.

148 "Tivey L., Interpretations of British Politics. The Image and the System ", Revue française de science politique, vol. 41, n6, 1991, p. 846-847.

149 «Goldring Maurice, Belfast from Loyalty to Rebellion », Vingtième Siècle, vol. 33, 1992, p. 1478.

150 « Du Déclin britannique en général et du thatchérisme en particulier. Capet Antoine, Le poids des années de guerre. Les classes dirigeantes britanniques et la réforme sociale 1931-1951; Farnetti Richard, Le Déclin de l'économie britannique de Victoria à Thatcher; Poirier François, Génération Thatcher. La Culture politique de l'Angleterre ", Revue française de science politique, vol. 43, $\mathrm{n}^{\circ} 1,1993$, p. 139-143.

151 Margaret Thatcher, Ten Downing Street, Quinzaine Littéraire, n637, décembre 1993.

152 «Foley Michael, The Rise of the British Presidency; Lenman Bruce, The Eclipse of Parliament. Appearance and Reality of British Politics since 1914 ; Judge David, The Parliamentary State ", Revue française de science politique, vol. 44, n 6, 1994.

153 «Crowe Sybil, Corp Edward, Our Ablest Public Servant Sir Eyre Crowe 1864-1925 », Vingtième Siècle, vol. 43, 1994, p. 150-151.

154 «Journès Claude, L'État britannique », Vingtième Siècle, vol. 48, 1995, p. 193-194. 
155 « À propos de la trilogie de Keith Middlemas État et société en Grande-Bretagne depuis 1940 ", Vingtième Siècle. Revue d'histoire, n49, 1996, p. 110-121.

"Hamer David, Can Responsible Government Survive in Australia? ", Vingtième Siècle, vol. 55, 1997, p. 189-190.

"Ouvrages récents autour de la question écossaise ", Revue française de civilisation britannique, vol. 9, n², mai 1997, p. 115-120.

158 «Crowley John, Sans épines, la rose. Tony Blair, un modèle pour l'Europe?", Revue française de science politique, vol.49, $\mathrm{n}^{\circ} 4,1999, \mathrm{p} .721-723$.

159 «Bédarida François, Churchill », Revue française de science politique, vol. 50, n6, 2000, p. 996-998.

\section{AUTEUR}

\section{EDWIGE CAMP-PIETRAIN}

Université Polytechnique des Hauts-de-France (Valenciennes) 